\title{
ANÁLISE BIBLIOGRÁFICA E MAPEAMENTO DA PRODUÇÃO DE UM GRUPO DE PESQUISA SOBRE O USO COMPETITIVO DA TECNOLOGIA DE INFORMAÇÃO
}

\author{
Heitor M. Quintella ${ }^{1}$ \\ Savio Domingos Coube Bogado ${ }^{2}$.
}

\begin{abstract}
Resumo: O artigo é baseado na análise bibliográfica que foi realizada paralelamente com a revisão de literatura da dissertação de mestrado em Engenharia de Produção "Estado da arte do uso competitivo de tecnologia de informação no setor de bebidas não-alcoólicas” (Bogado, 2003). Esta dissertação é parte integrante da pesquisa da UFF sobre Fatores Humanos e Tecnológicos da Competitividade (FHTC) que, anteriormente, pesquisou os segmentos de varejo, de produtos médicos e de telecomunicações. A análise bibliográfica baseou-se em 39 obras da bibliografia da dissertação, selecionadas pelo seu alinhamento com o tema do uso competitivo da tecnologia de informação para a industria em geral e, não somente, para a industria de bebidas não-alcoólicas.Os resultados da análise bibliográfica tem uma contribuição importante, tanto para o grupo FHTC quanto para outros grupos que pesquisem temas correlatos, oferecendo subsídios para a análise, correções e aperfeiçoamentos dos rumos intelectuais das pesquisas. Objetivou-se, portanto, criar um referencial para o meio acadêmico, consultores de empresas e profissionais, sobre as tendências de uso de autores, editoras, revistas e periódicos especializados que abordam os temas Competitividade e Uso competitivo da tecnologia de informação.
\end{abstract}

Palavras-chave: Competitividade, Tecnologia de Informação, Estratégia competitiva.

Abstract: The article is based on the analysis of the bibliography that has been conducted in parallel with the literature revision of the Production Engineering master thesis "The state of art of the information technology competitive use in the non-alcoholic beverage industry" (Bogado, 2003). This thesis is part of the Universidade Federal Fluminense (UFF) Human and Technologic Factors of the Competitiveness (HTFC) Project that previously has searched the retail, the medical products and the telecommunication industries. The analysis is based on 39 works included in the Bibliography chapter of the referenced thesis and they were selected due to their content alignment with the competitive use of information technology in the industry as a whole and not only in the non-alcoholic beverage industry. The results of this bibliography analysis are of a great contribution both to the HTFC research group and to the other research groups which are working in similar matters, offering as benefits the analysis, corrections and improvements of the intellectual directions of these researches. The main objective of this work is to create a reference to the academic environment, enterprise consultants and professionals which are dealing with competitive use of information technology, helping them to select the state of art of authors, publishing companies and specialized magazines.

Key-words: Competitiveness, Information Technology, Competitive Strategy.

\footnotetext{
${ }^{1}$ Universidade Federal Fluminense

${ }^{2}$ LEXSAR - Comércio, Serviços e Logística Ltda
} 


\section{INTRODUÇÃO}

No capitulo de revisão de literatura das teses e dissertações de mestrado e doutorado, normalmente, encontra-se comparações entre o tema que está sendo pesquisado e os temas correlatos contidos em trabalhos de outros autores.

Para Luna (1997), a revisão de literatura em um trabalho de pesquisa pode ser realizada com os objetivos de:

a) determinar o "estado da arte", procurando mostrar através da literatura existente o que já se sabe sobre o tema, as lacunas existentes e os principais entraves teóricos ou metodológicos;

b) fazer uma revisão teórica, visando inserir o problema da pesquisa dentro de um quadro de referência teórica para explicá-lo;

c) fazer uma revisão empírica, onde procura-se explicar a pesquisa do ponto de vista metodológico, buscando-se respostas para questões como: quais os procedimentos normalmente empregados em pesquisas similares? Que fatores afetaram os resultados? Que propostas têm sido feitas para analisá-los, explicálos ou controla-los? Como foram analisados os resultados?

d) fazer uma revisão histórica, quando se busca a evolução de conceitos, temas e outros aspectos que tenham correlação com o problema da pesquisa, fazendo a inserção dessa evolução dentro de quadro teórico de referência que explique os fatores determinantes e as implicações das mudanças.

$\mathrm{Na}$ pesquisa "Estado da arte do uso competitivo de Tecnologia de Informação no Setor de bebidas nãoalcoólicas”, fomos além dos objetivos elencados por Luna (1997) para uma revisão de literatura, ao incorporar uma análise bibliográfica sobre o uso de autores, revistas especializadas e editoras dentro dos temas pesquisados, competitividade e tecnologia de informação.

\section{OBJETIVOS DA ANÁLISE BIBLIOGRÁFICA}

Os principais objetivos da análise bibliográfica são:

a) analisar o "estado da arte" da literatura que está sendo utilizada em trabalhos que abrangem os temas competitividade e tecnologia de informação como alavancadora de vantagem competitiva sustentável, aí incluídos teses e dissertações, trabalhos apresentados e publicados em anais de congressos e outros encontros científicos, artigos publicados em revistas ou periódicos e livros;

b) delimitar o período de tempo em que estas obras foram publicadas;

c) verificar a existência de unanimidade na citação de autor(es), livro(s), revista(s), editora(s), encontro(s) científico(s), o que dará uma indicação de fontes de informação de prestígio onde pode-se pesquisar trabalhos e publicar artigos;

d) ser um referencial bibliográfico para o meio acadêmico e pessoas interessadas nestes assuntos.

\section{METODOLOGIA UTILIZADA}

Selecionou-se os trabalhos analisados em quatro categorias: teses e dissertações de mestrado e doutorado, artigos apresentados em encontros científicos, artigos publicados em revistas e periódicos especializados e livros.

Dentro de cada uma destas quatro categorias foi analisada a bibliografia de cada trabalho, grupando as obras referenciadas, também, nas quatro categorias acima descritas. Por exemplo, dentro da capítulo bibliografia de uma tese, quais e quantas obras listadas eram teses , artigos apresentados em encontros científicos, artigos publicados em revistas e livros.

Em seguida, identificou-se os autores, os eventos, as revistas, as editoras e o ano de publicação visando identificar o que era comum a todos ou a maior parte dos trabalhos selecionados para esta análise bibliográfica. 
As principais palavras-chave da pesquisa eram: competitividade e tecnologia de informação utilizadas separadamente e combinadas numa triagem final.

\section{A AMOSTRA DA ANÁLISE BIBLIOGRÁFICA}

Buscou-se obras que focalizassem o uso da tecnologia de informação como alavancadora de ganho de competitividade sustentável pelos diversos segmentos da indústria. Um dos caminhos de busca foi a Internet. Utilizou-se o argumento de pesquisa competitividade e observou-se uma grande quantidade de obras em universidades de prestígio como a UFRJ, UFF, UFSC, USP, UFRGS, UNICAMP, UFPE, UFPR, PUC/Rio, PUC/Paraná e PUC/RGS. Visitou-se, também, as bibliotecas da COPPE e COPPEAD (UFRJ), da UFF, da PUC/Rio e da FGV/Rio e igualmente encontrou-se diversos trabalhos sobre competitividade. Contudo, quando associou-se o argumento de pesquisa competitividade ao argumento tecnologia de informação, este universo foi significativamente reduzido.

Considerando-se a complexidade em se determinar uma amostra estatística de um universo bastante difícil de se definir, procurou-se selecionar obras que, na opinião dos autores, estivessem plenamente alinhadas com o tema em análise e com o referencial teórico da linha de pesquisa do projeto FHTC e que propiciassem resultados confiáveis para o trabalho.

Identificou-se cerca de uma centena de obras, nas quatro categorias, de onde extraiu-se 39 obras, como detalhadas no Quadro 1 :

\begin{tabular}{|c|c|c|}
\hline CATEGORIAS & $\begin{array}{c}\text { NÚMERO DE } \\
\text { TRABALHOS }\end{array}$ & $\begin{array}{c}\text { NÚMERO } \\
\text { DE } \\
\text { AUTORES }\end{array}$ \\
\hline $\begin{array}{c}\text { TESES E } \\
\text { DISSERTAÇÕES }\end{array}$ & 7 & 7 \\
\hline $\begin{array}{c}\text { ARTIGOS DE } \\
\text { EVENTOS } \\
\text { CIENTÍFICOS }\end{array}$ & 12 & 20 \\
\hline $\begin{array}{c}\text { ARTIGOS DE } \\
\text { REVISTAS }\end{array}$ & 15 & 17 \\
\hline LIVROS & 5 & 3 \\
\hline TOTAL & 39 & 47 \\
\hline
\end{tabular}

Quadro1- Amostra da análise bibliográfica Fonte: Autores do artigo (2003)

A análise do capítulo bibliografia dos 39 trabalhos selecionados para amostra, como grupados no Quadro 1 acima, relacionou algumas centenas de títulos e autores. Somente numa tese de doutorado, constatou-se a existência de 26 páginas de referências bibliográficas.

Para que os resultados tivessem maior significância, já que buscava-se conhecer o "estado da arte" e as tendências das bibliografias utilizadas pelos autores das 39 obras, adotou-se como linha de corte, as citações que tivessem 3 ou mais referências nas bibliografias de mais de um trabalho, ou seja, desconsiderou-se os autores, livros e revistas que apareceram menos de 3 vezes e somente em um trabalho da amostra pesquisada.

Os resultados encontrados estão sumarizados, por cada um dos quatro grupos pesquisados, nos itens 6, 7, 8 e 9 à seguir.

\section{REFERENCIAL TEÓRICO}

O referencial teórico do projeto de pesquisa FHTC da UFF com o qual este trabalho está alinhado é composto pelo modelo de Estabilidade dinâmica de Joseph Pine (1994) e pelo modelo da Cadeia de valor de Michael Porter (1989).

Segundo Pine, o novo paradigma de administração que hoje está emergindo em substituição ao sistema de produção em 
massa que vigorou por muitas décadas e ainda é utilizado por um número significativo de empresas é a customização maciça. Neste novo sistema, os processos são mais importantes do que os produtos e os consumidores em mercados heterogêneos crescentes demandam produtos personalizados. A turbulência no mercado exige que as empresas tenham o que Pine (1994) definiu como estabilidade dinâmica, ou seja processos robustos e estáveis que suportem a dinâmica de mudanças nos produtos para atender as necessidades e exigências dos clientes, como representado no Quadro 2 :

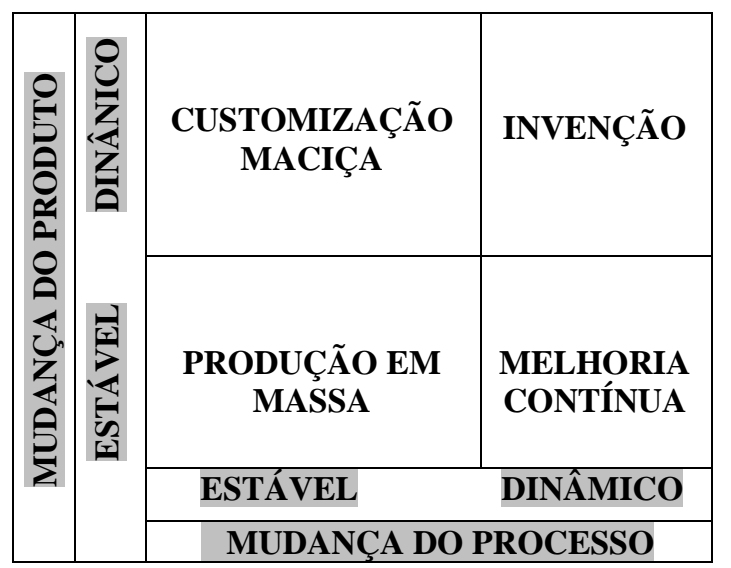

Quadro 2: Modelo de estabilidade dinâmica Fonte: Pine (1994)

O modelo da Cadeia de valor de Porter(1989), apesar de publicado há mais de 15 anos, permanece atual e largamente utilizado por consultores em estratégia empresarial. A Cadeia de valor de toda empresa, segundo Porter, é composta de nove categorias genéricas de atividades agrupadas em dois tipos de atividades: as atividades primárias (logística interna, operações, logística externa, marketing e vendas, serviços pós-venda) e as atividades de apoio (infraestrutura, recursos humanos, desenvolvimento e tecnologia, aquisição/compras), como ilustrado no Quadro 3. A tecnologia de informação permeia todos os pontos da Cadeia de valor, transformando o modo como as atividades são realizadas e, com isto, alterando o escopo da competitividade e a maneira como os produtos e serviços atendem as necessidades dos consumidores.

\begin{tabular}{|c|c|c|c|c|}
\hline \multicolumn{5}{|c|}{ ATIVIDADES DE APOIO } \\
\hline \multicolumn{5}{|c|}{ INFRA-ESTRUTURA } \\
\hline \multicolumn{5}{|c|}{ RECURSOS HUMANOS } \\
\hline \multicolumn{5}{|c|}{ DESENVOLV.TECNOLOGIA } \\
\hline \multicolumn{5}{|c|}{ AQUISIÇÃO/COMPRAS } \\
\hline 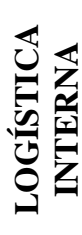 & 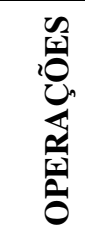 & 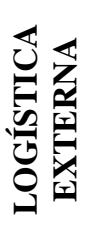 & 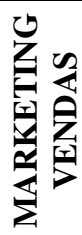 & 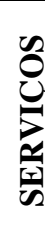 \\
\hline
\end{tabular}

ATIVIDADES PRIMÁRIAS

Quadro 3: Modelo da Cadeia de Valor Fonte: Porter (1989)

\section{ANÁLISE DAS TESES E DISSERTAÇÕES DE MESTRADO E DOUTORADO}

A amostra pesquisada foi de 7 teses e dissertações das universidades UFF, UFRJ (COPPEAD) e UFSC.

A universidade mais referenciada nestes trabalhos é a USP.

Os artigos mais comumente utilizados são os apresentados no ENANPAD (Encontro Nacional da Associação Nacional dos Programas de Pós-Graduação em Administração) e do ENEGEP (Encontro Nacional de Engenharia de Produção).

Observou-se, também, referências a eventos internacionais como: Annual Conference on Information Systems AIS/ICIS (USA), International Conference on Systems Sciences (USA) e International Conference on Management Technology.

\subsection{REVISTAS}

Os artigos publicados em revistas técnicas e especializadas, especialmente as internacionais, são largamente utilizados como fonte de consulta nas teses e dissertações analisadas, representando o maior percentual do conteúdo das bibliografias.

HARVARD BUSINESS REVIEW é a revista mais referenciada, seguindo-se MIT SLOAN MANAGEMENT REVIEW, MIS QUARTERLY, JOURNAL OF MANAGEMENT 
INFORMATION SYSTEMS, HSM

MANAGEMENT, EXAME, CONJUNTURA ECONÔMICA, IBM

SYSTEMS JOURNAL, DECISION

SCIENCES, COMPUTERWORLD, REVISTA DE ADMINISTRAÇÃO DE

EMPRESAS, ADMINISTRATIVE

SCIENCE QUARTERLY,

INFORMATION SYSTEMS

RESEARCH, STRATEGIC

MANAGEMENT JOURNAL e THE

JOURNAL OF BUSINESS

STRATEGY.

\subsection{AUTORES}

MICHAEL PORTER está presente, com um ou mais títulos, nas bibliografias das sete teses e dissertações analisadas, ratificando a importância deste autor quando o tema é competitividade empresarial.

Outros autores que aparecem com freqüência são: PINE,J.; LAKATOS, E.M.; MARCONI, M.; CHIAVENATO, I. e YIN, R.K.

\subsection{EDITORAS}

Diversos trabalhos publicados pela ATLAS constam de todas as bibliografias.

Outras editoras citadas com freqüência são: CAMPUS, MAKRON BOOKS, JOHN WILEY \& SONS, MC GRAWHILL, HARVARD BUSINESS SCHOOL PRESS, OXFORD UNIVERSITY PRESS e PRENTICEHALL.

\section{ANÁLISE DE ARTIGOS APRESENTADOS EM ENCONTROS CIENTÍFICOS}

Pesquisou-se 12 artigos, sendo 11 de autores brasileiros e apresentados no ENANPAD e no ENEGEP e 1 de autores alemães, apresentado no ASAC-IFSAM CONFERENCE, Montreal, Canadá (2000). Procurou-se analisar artigos do ENANPAD e do ENEGEP posteriores a 1997 à partir de quando os critérios de seleção tornaramse mais rigorosos e os trabalhos foram disponibilizados em CD’s, o que facilita a pesquisa.

\subsection{REVISTAS}

As revistas mais referenciadas nos doze trabalhos analisados são: HARVARD BUSINESS REVIEW, SLOAN MANAGEMENT REVIEW, EXAME, IBM SYSTEMS JOURNAL, STRATEGIC MANAGEMENT JOURNAL e EUROPEAN JOURNAL OF INFORMATION SYSTEMS.

\subsection{AUTORES}

Os autores mais citados são: PORTER, M.; QUINTELLA, H.M.; VENKATRAMAN, N. e HENDERSON, J.C.

\subsection{EDITORAS}

As editoras mais referenciadas nos trabalhos apresentados em Encontros Científicos são: CAMPUS, ATLAS, MAKRON BOOKS, THE FREE-PRESS, HARVARD BUSINESS SCHOOL PRESS, BOOKMAN e PRENTICEHALL.

\section{ANÁLISE DE ARTIGOS PUBLICADOS EM REVISTAS TÉCNICAS}

Pesquisou-se 15 artigos, sendo 10 de autores brasileiros e 5 de autores estrangeiros.

\subsection{REVISTAS}

As mais citadas são HARVARD BUSINESS REVIEW e STRATEGIC MANAGEMENT JOURNAL

\subsection{AUTORES}

Existe uma grande dispersão de autores citados nas bibliografias mas, como nas demais categorias, MICHAEL PORTER é o referenciado com maior freqüência.

\subsection{EDITORAS}

As editoras mais utilizadas são THE FREE PRESS, CAMPUS e HARVARD BUSINESS SCHOOL PRESS. 


\section{ANÁLISE DE LIVROS}

Escolheu-se na Amostra cinco livros cujos temas estão plenamente sintonizados com Competitividade e com o uso da TI como alavancadora de vantagem competitiva sustentável. São eles: "Made in Brazil" (Ferraz, J.C.; Kupfer, D.; Haguenauer, L.), "A Estratégia e o cenário dos negócios” (Ghemawat, P.), "Estratégia Competitiva: Técnicas para Análise de Industrias e da Concorrência” (Porter, M.), "Vantagem Competitiva: Criando e Sustentando um Desempenho Superior" (Porter, M.) e "Personalizando Produtos e Serviços-Customização Maciça” (Pine, J.).

\subsection{REVISTAS}

As revistas mais referenciadas pelos autores dos livros analisados são: HARVARD BUSINESS REVIEW, CALIFORNIA MANAGEMENT REVIEW e JOURNAL OF MARKETING.

\subsection{AUTORES}

MICHAEL PORTER é referenciado pelos outros autores pesquisados. Destacam-se, também: ABERNATHY, W.J.; BUZZELL, R.D.; LEVITT, T. e CAVES, R.E.

\subsection{EDITORAS}

As editoras mais citadas são: HARVARD SCHOOL OF BUSINESS ADMINISTRATION, THE FREE PRESS, PRENTICE-HALL, HARVARD UNIVERSITY PRESS E MC GRAWHILL.

\section{PONTOS IMPORTANTES SOBRE O USO COMPETITIVO DA TI}

Encontrou-se diversos pontos nos trabalhos analisados que ratificam a importância do uso competitivo da tecnologia de informação (TI). Dentre eles, destacam-se os seguintes:

- Existe um enorme potencial de uso da TI "extra-muros" das empresas, ou seja, a TI está sendo utilizada maciçamente para solução dos processos internos e, timidamente, para os processos da cadeia de valor com envolvimento dos fornecedores, canais de distribuição e clientes/consumidores finais. (DANTAS, Eduardo. 2000)

- A TI é a única alternativa para racionalizar o fluxo de informações cada vez maior e mais complexo dentro da cadeia de valor das empresas. (FERRAZ, J.C; KUPFER, D.; HAGUENAUER, L. 1997)

- Cada empresa deve escolher a tecnologia mais adequada ao seu perfil competitivo e, não, o que existe de mais moderno e mais caro no mercado. (FREITAS, H. et al. 2002)

- A estratégia de TI deve estar, permanentemente, alinhada com as estratégias da empresa visando não só obter vantagem competitiva mas a perenidade da empresa. (FREITAS, $\mathrm{H}$. et al.2002)

- Começar do zero na TI pode não ser uma desvantagem. Como o mundo dos negócios continuará sendo turbulento através da globalização, do aumento da competição e do desenvolvimento tecnológico, as empresas terão que repensar, continuadamente, as suas práticas de negócios, possibilitando aos novos entrantes alinharem-se, à qualquer momento, com o "estado da arte” da TI. (FREITAS, H. et al. 2002)

- As empresas bem sucedidas reverão, permanentemente, a forma de operar os seus negócios. Seus gerentes colocar-se-ão como agentes de mudança ao invés de agirem reativamente e os executivos conhecerão, profundamente, os pontos fortes e fracos da TI, melhor utilizando-a para ganhar vantagem competitiva. (CANE, A . 1992)

- Considerando que os fatores humanos são e serão os elementos mais importantes para o sucesso do uso da TI numa organização, todo o investimento em educação, em instituições formais ou outras formas 
de treinamento, terá alto retorno. (CANE, A .1992)

- Adaptando-se a influência do modelo das cinco forças competitivas de PORTER e MILLAR (1985) à competitividade das empresas, a TI tem os seguintes impactos:

CLIENTES: a TI pode contribuir para a redução do poder de barganha dos clientes na competitividade das empresas, fornecendo benefícios no processo de comunicação, nos produtos e nos serviços que os mantenham leais às empresas;

FORNECEDORES: a TI pode impor mecanismos de relação das empresas com seus fornecedores e antecipar mudanças nos produtos, custos e processos dos fornecedores que poderão impactar a sua competitividade em relação às demais empresas do seu segmento industrial;

CONCORRENTES: a TI ao aumentar o vínculo da empresa com seus clientes e fornecedores, propicia que ela não fique em desvantagem em relação aos seus competidores;

NOVOS ENTRANTES: o forte relacionamento entre a empresa e seus clientes e fornecedores gerado pela TI pode inibir a entrada de novos concorrentes no seu segmento de mercado; PRODUTOS SUBSTITUTOS: a TI aо identificar as ameaças do surgimento de novos produtos que aumentem o leque de opções para os clientes, favorece ações rápidas da empresa para que se antecipe aos concorrentes e, mesmo, estabeleça alianças com os concorrentes ameaçadores. (CORNELLA, A .1994)

\section{CONCLUSÕES}

As principais conclusões da análise bibliográfica das 39 obras são:

\subsection{AUTORES}

MICHAEL PORTER foi referenciado 69 vezes nas quatro categorias analisadas, mostrando a importância deste autor no meio científico e acadêmico.

Com menor freqüência de referências, mas presentes nas bibliografias das quatro categorias analisadas, encontramse: HAMEL, G.; HENDERSON, J.C.; LUFTMAN, J.N.; MINTZBERG, H.; PRAHALAD, C.K.; PINE, J.B. e VENKATRAMAN, N., como mostrado no Gráfico 1:

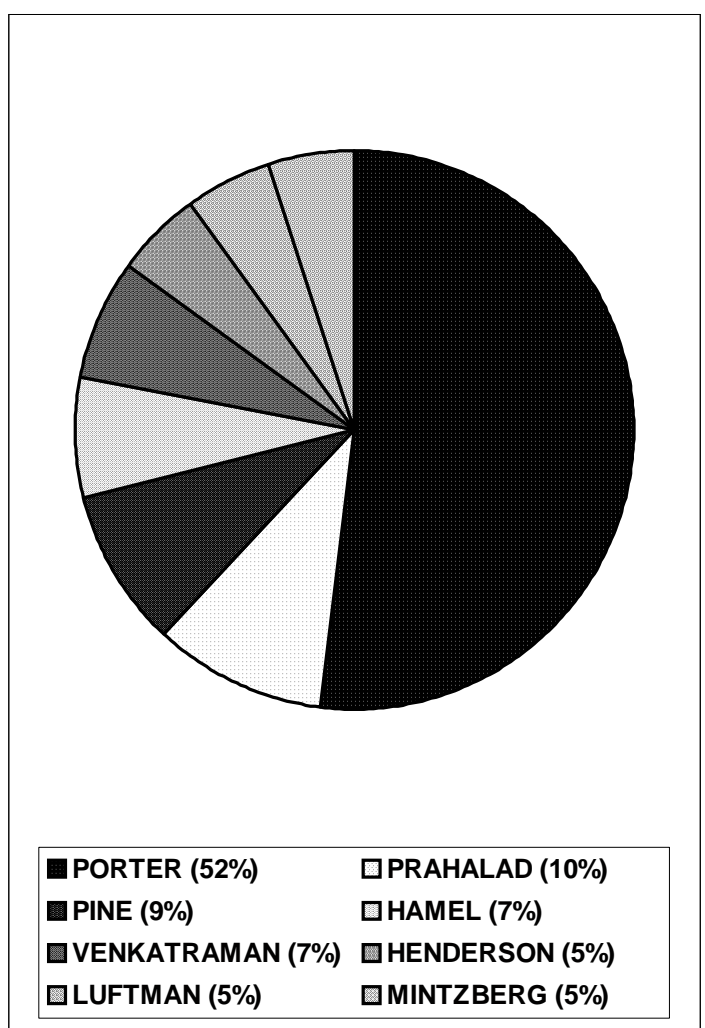

Gráfico 1 - Autores mais referenciados na análise bibliográfica

Fonte: Autores do artigo (2003)

\subsection{REVISTAS}

As revistas mais referenciadas como mostrado no Gráfico 2 foram: HARVARD BUSINESS REVIEW (USA); STRATEGIC MANAGEMENT JOURNAL (USA); MIT SLOAN MANAGEMENT REVIEW (USA); EXAME (Brasil); IBM SYSTEMS JOURNAL (USA); HSM MANAGEMENT (Brasil);

CALIFORNIA MANAGEMENT REVIEW (USA) e JOURNAL OF MARKETING (USA).

Citadas com menor freqüência, mas que devem ser consideradas quando se pensar em publicar artigos em revistas internacionais, destacam-se: MIS 
QUARTERLY (USA), JOURNAL OF MANAGEMENT (USA), DECISION SCIENCES (USA), THE JOURNAL OF BUSINESS STRATEGY (USA), e EUROPEAN JOURNAL OF INFORMATION SYSTEMS

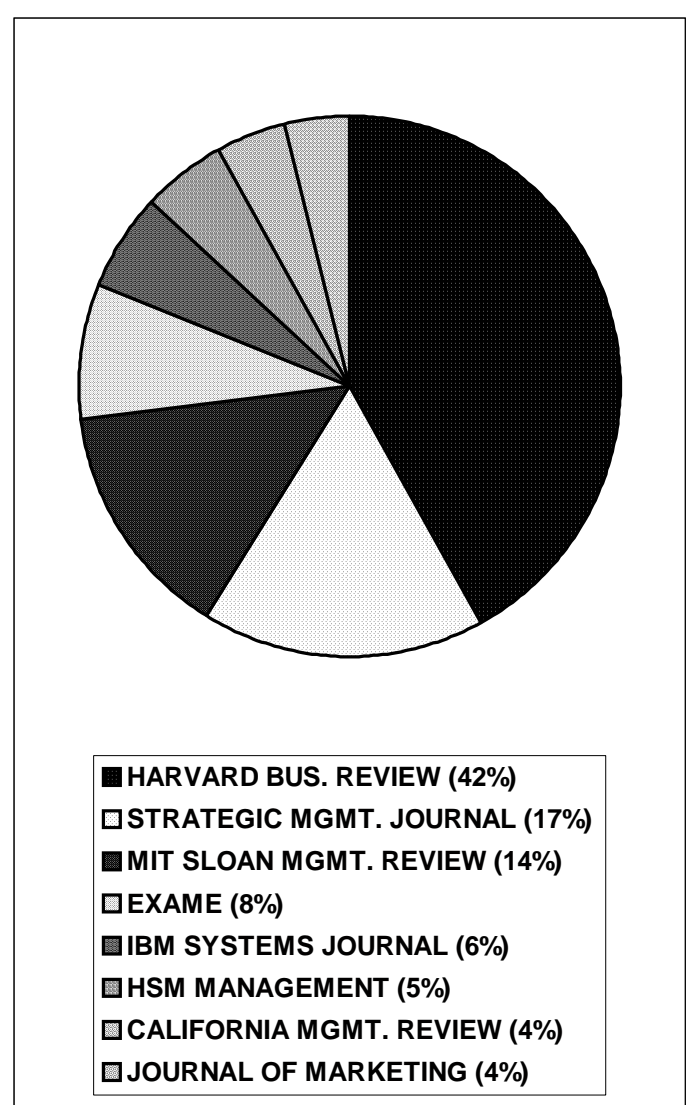

Gráfico 2 - Revistas mais referenciadas na análise bibliográfica

Fonte: Autores do artigo (2003)

\subsection{EDITORAS}

As editoras mais citadas foram: CAMPUS (Brasil); ATLAS (Brasil); THE FREE PRESS (USA); MAKRON BOOKS (Brasil); HARVARD BUSINESS SCHOOL PRESS (USA); JOHN WILEY \& SONS (USA); McGRAW-HILL (USA); PRENTICEHALL (USA) e HARVARD UNIVERSITY PRESS USA), como mostrado no Gráfico3 abaixo:

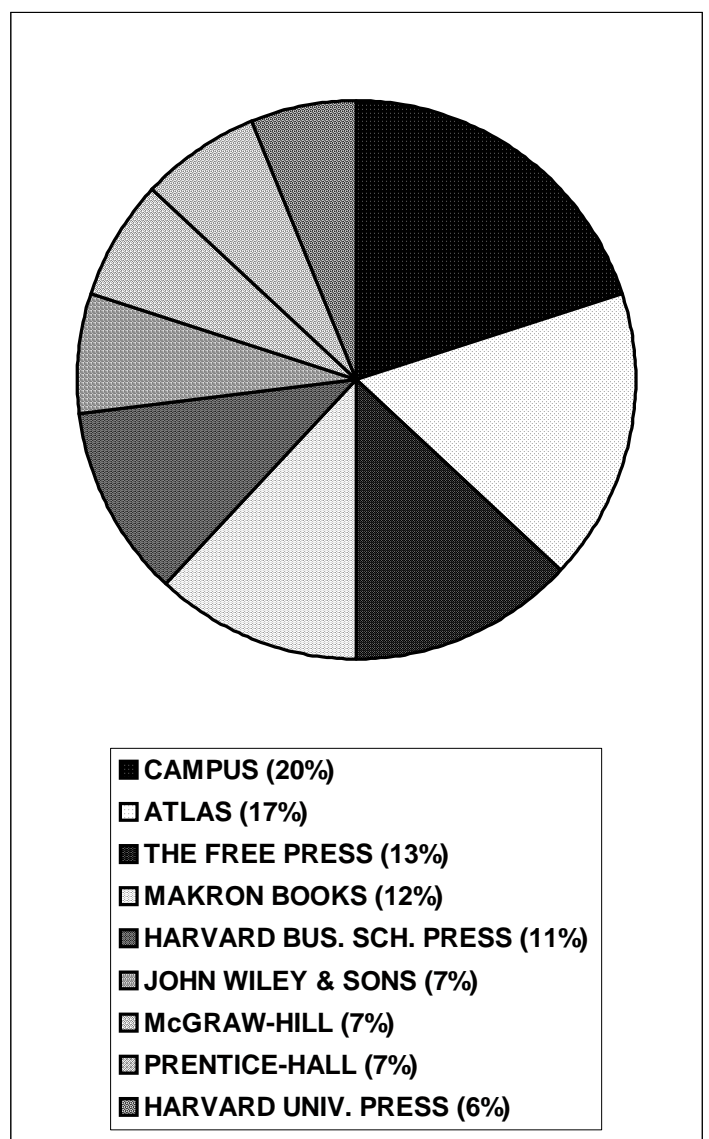

Gráfico 3 - Editoras mais referenciadas na análise bibliográfica

Fonte: Autores do artigo (2003)

\subsection{OUTRAS CONCLUSÕES}

O período de publicação das obras referenciadas nas 39 bibliografias analisadas está compreendido entre 1937 e 2002, com diversas citações das décadas de 50, 60, 70 e 80, especialmente esta última.

A grande maioria dos autores referenciados é de estrangeiros (mais de 90\% dos nomes analisados).

As bibliografias dos trabalhos de autores estrangeiros, particularmente os artigos apresentados em eventos científicos e os publicados em revistas especializadas, apresentam um número maior de referências do que as dos autores brasileiros.

A universidade de Harvard (USA) é a que aparece com maior destaque entre as universidades estrangeiras

No Brasil, destacam-se a USP, a UFRJ, a UFRGS, a UFSC e a FGV. 


\section{BIBLIOGRAFIA}

\subsection{AMOSTRA ANALISADA}

ABREU, Aline F.; FRANÇA, Thamara C.V.; SINZATO, Carmen I.P. Acesso à informação - promovendo competitividade em P\&D com o uso de tecnologia de informação.CIÊNCIA DA INFORMAÇÃO, Brasília, v.28, n.3, pp.322-332, set./dez. 1999.

BARBOSA Jr., Luiz P.; MORAES, Walter F.A . Estratégias em ambientes hipercompetitivos: $O$ caso da indústria brasileira de embalagens para cervejas $e$ refrigerantes. In: ENANPAD ENCONTRO NACIONAL DA ASSOCIAÇÃO NACIONAL DOS PROGRAMAS DE PÓS-GRADUAÇÃO EM ADMINISTRAÇÃO, XXV. 2001, Campinas. Anais do ENANPAD/2001, 15f., ESO 276.

BETTIS, Richard A.;HITT, Michael A. The new competitive lanscape.STRATEGIC MANAGEMENT JOURNAL, Illinois/USA, v.16, Summer Special Issue Series, pp.7-19, 1995.

BLACK, Janice A.; BOAL, Kimberly B. Strategic Resources: Traits, Configurations and Paths to sustainable competitive advantage.

STRATEGIC MANAGEMENT JOURNAL, Illinois/USA, v.15, pp.131-148, 1994.

BUIAR, Denise R. Vantagem Competitiva da Flexibilidade via Tecnologia de Informação: Um modelo de Auditoria e Estudo de Caso no Pólo Automotivo Paranaense.2000. 218 f. Tese (Doutorado em Engenharia de Produção) Departamento de Engenharia de Produção e Sistemas, Universidade Federal de Santa Catarina, Florianópolis, 2000.

CANE, Alan. Information Technology and Competitive Advantage: Lessons from the Developed Countries .WORLD VELOPMENT, Inglaterra, v.20, n.12, p. 1721-1736, 1992.

CARVALHO, Jaci, T.O. A Competitividade das Industrias de Produtos Médicos: Uma análise à luz dos ENGEVISTA, v. 6, n. 2, p. 36-47, agosto. 2004
Modelos de Estabilidade Dinâmica e Cadeia de Valor. 2001. 148 f. Dissertação (Mestrado em Engenharia de Produção) Centro Tecnológico, Curso de PósGraduação em Engenharia de Produção, Universidade Federal Fluminense, Niterói. 2001.

COSTA, Luis Guilherme R.M. Mercado de Refrigerantes Brasileiro: Uma Análise do crescimento das marcas populares. 2001. 91f. Dissertação (Mestrado em Administração) - COPPEAD - Instituto de Pós-Graduação e Pesquisa em Administração, Universidade Federal do Rio de Janeiro, Rio de Janeiro. 2001.

DANTAS, Eduardo M.A .Estágio da Organização Logística de três empresas do setor de bebidas: um Estudo de Caso. 2000. 145 f. Dissertação (Mestrado em Administração) - COPPEAD - Instituto de Pós-Graduação e Pesquisa em Administração, Universidade Federal do Rio de Janeiro, Rio de Janeiro. 2000.

DiSERIO, Luiz Carlos; MARQUES, Érico V.; MAGNANI, Alexandre. The use of Information Technology as Factor of Competitiveness in Brazilian Retail. BALAS - THE BUSINESS ASSOCIATION OF LATIN AMERICAN STUDIES, Caracas/Venezuela, 9f., 2001.

FERRAZ, João Carlos; KUPFER, David; HAGUENAUER, Lia. Made in Brazil: Desafios Competitivos para a indústria. 3. ed. Rio de Janeiro: Campus, 1997. 386p.

FREITAS, Henrique et al. Competitividade na Era da Informação e da Internet: Estudo Exploratório com Executivos. In: ENANPAD - ENCONTRO NACIONAL DA ASSOCIAÇÃO DOS PROGRAMAS DE PÓS-GRADUAÇÃO EM ADMINISTRAÇÃO, XXVI. 2002, Salvador. Anais do ENANPAD/2002, 15f., ADI 1520.

GHEMAWAT, Pankaj. A Estratégia e o cenário dos negócios, Texto e casos.Porto Alegre: Bookman, 2000.

GRAÇA, Carolina T.; Sobral, Maria Cecília; ANEFALOS, Lílian Cristina. 
Novo Paradigma Brasileiro no Setor de Bebidas: o caso AMBEV. In: ENANPAD ENCONTRO NACIONAL DA ASSOCIAÇÃO NACIONAL DOS PROGRAMAS DE PÓS-GRADUAÇÃO EM ADMINISTRAÇÃO, XXVI. 2002, Salvador. Anais do ENANPAD/2002, 16f., GOL 1831.

LAURINDO, Fernando J.B. Tecnologia de Informação como suporte às estratégias empresariais. In: ENEGEP - ENCONTRO NACIONAL DE ENGENHARIA DE PRODUÇÃO, XVIII. 1998, Niterói. Anais do ENEGEP/1998, $14 f$.

LOEBBECKE, Claudia; POWELL, Philip. Competitive Advantage from IT in Logistics: The Integrated Transport Tracking System. INTERNATIONAL JOURNAL OF INFORMATION MANAGEMENT, Inglaterra, v.18, n.1, p. 17-27, 1998.

MAIA, Marta C.; DiSERIO, Luiz Carlos; CAMPOS, Ricardo J.F. Tecnologia para integração de Projetos de Produtos $e$ Processos. In: ENEGEP - ENCONTRO NACIONAL DE ENGENHARIA DE PRODUÇÃO, XX. 2000, São Paulo. Anais do ENEGEP/2000, $13 f$.

OLIVEIRA, Luiz Carlos A. et al. A Tecnologia da Informação como instrumento do posicionamento estratégico. In: ENANPAD ENCONTRO NACIONAL DA ASSOCIAÇÃO NACIONAL DOS PROGRAMAS DE PÓS-GRADUAÇÃO EM ADMINISTRAÇÃO, XXV. 2001, Campinas. Anais do ENANPAD/2001, 15f., ESO 1236.

PAIM, Dario J.B. Indústria de Telecomunicações, uma Analise de Competitividade à luz dos Modelos de Estabilidade Dinâmica e Cadeia de Valor. 2002. 127 f. Dissertação (Mestrado em Sistemas de Gestão) - Centro Tecnológico, LATEC, Universidade Federal Fluminense, Niterói. 2002.

PILLER, Frank T.; REICHWALD, Ralf; MÖSLEIN, Kathrin. Information as a critical success factor for mass customization or: why even a customized shoe not always fits.In: ASAC - IFSAM CONFERENCE. 2000, Montreal/Canada. Technische Universität München, Germany, 10f.

PINE II, Joseph. Personalizando Produtos e Serviços - Customização Maciça - A Nova Fronteira da Competição dos negócios.São Paulo: Makron Books, 1994.

PORTER, Michael E. Estratégia Competitiva: Técnicas para análise de indústrias e da concorrência. 7. ed. Rio de Janeiro: Campus, 1986.

PORTER, Michael E. Vantagem Competitiva, criando e sustentando um desempenho superior.13. ed. Rio de Janeiro: Campus, 1989.

PORTER, Michael E. Towards a dynamic theory of strategy. STRATEGIC MANAGEMENT JOURNAL, Illinois/USA, v. 12, p. 95-117, 1991.

QUINTELLA, Heitor L.M.M. Tecnologia de Informação no Modelo de Estabilidade Dinâmica. In: ENEGEP - ENCONTRO NACIONAL DE ENGENHARIA DE PRODUÇÃO, XVII. 1997, Gramado. Anais do ENEGEP/1997, 7f.

QUINTELLA, Heitor L.M.M. Tecnologia de Informação $e$ avaliação da competitividade no Brasil. SUMA ECONÔMICA, Rio de Janeiro, Agosto, pp. 46-47, 1998.

QUINTELLA, Heitor L.M.M. Competitividade e Tecnologia de Informação - Análise de Empresas com tendências à customização em massa. SUMA ECONÔMICA, Rio de Janeiro.Abril, pp. 42-43, 2000.

QUINTELLA, Heitor L.M.M.; CARVALHO, Jaci T.O . A Tecnologia da Informação $e$ a Competitividade na Industria de Produtos Médicos. REVISTA TENDÊNCIAS DO TRABALHO, Rio de Janeiro, Março, pp.14-21, 2002.

QUINTELLA, Heitor L.M.M.; Carvalho, Jaci T.O . A Tecnologia da Informação e a 
Competitividade na Industria de Produtos Médicos. In: ENANPAD - ENCONTRO NACIONAL DA ASSOCIAÇÃO NACIONAL DOS PROGRAMAS DE PÓS-GRADUAÇÃO EM ADMINISTRAÇÃO, XXVI. 2002, Salvador. Anais do ENANPAD/2002, 9f, ADI 1043.

QUINTELLA, Heitor L.M.M.; COSTA, Sergio G. A Informática e a mudança do Paradigma Competitivo. CONJUNTURA ECONÔMICA/IBRE/FGV, v.51, pp. 3438, 1997.

QUINTELLA, Heitor L.M.M.; PAIM, Dario J.B. Tecnologia da Informação: ferramentas de competitividade. REVISTA TENDÊNCIAS DO TRABALHO, Rio de Janeiro, Julho, pp. 28-34, 2002.

QUINTELLA, Heitor L.M.M.; PAIM, Dario J.B. Tecnologia da Informação $e$ Customização em Massa no Setor de Telecomunicações. In: ENEGEP ENCONTRO NACIONAL DE ENGENHARIA DE PRODUÇÃO, XXII. 2002, Curitiba. Anais do ENEGEP/2002, $8 f$.

QUINTELLA, Heitor L.M.M.; SILVA, Luis Roberto. Customização em massa no varejo. REVISTA TENDÊNCIAS DO TRABALHO, Rio de Janeiro, Abril, pp.47, 2001.

QUINTELLA, Heitor L.M.M.; TEIXEIRA, Filho J. Internet $e$ Competitividade. REVISTA TENDÊNCIAS DO TRABALHO, Rio de Janeiro, Novembro, pp.24-27, 1999.

QUINTELLA, Heitor L.M.M.; ZANCA, J.F. Competitividade e Tecnologia da Informação - Setor de Alimentos $e$ Bebidas. REVISTA TENDÊNCIAS DO TRABALHO, Rio de Janeiro, Janeiro, pp. 28-34, 2002.

RESENDE, Paulo T.V. Estratégias Corporativas de reposicionamento na Cadeia de valor. In: ENANPAD ENCONTRO NACIONAL DA ASSOCIAÇÃO NACIONAL DOS PROGRAMAS DE PÓS-GRADUAÇÃO ENGEVISTA, v. 6, n. 2, p. 36-47, agosto. 2004
EM ADMINISTRAÇÃO, XXV. 2001, Campinas. Anais do ENANPAD/2001, 15f., ESO 696.

REZENDE, Denis. Alinhamento do Planejamento Estratégico da Tecnologia da Informação ao Planejamento Empresarial: proposta de um modelo $e$ verificação da prática em grandes empresas brasileiras. 2002. 279 f. Tese (Doutorado em Engenharia de Produção) Departamento de Engenharia de Produção e Sistemas, Universidade Federal de Santa Catarina, Florianópolis. 2002.

SANTOS, Selma R.S.; AZEVEDO, Paulo F. Concorrência no Mercado de Refrigerantes: impactos das novas embalagens. In: ENEGEP - ENCONTRO NACIONAL DE ENGENHARIA DE PRODUÇÃO, XX. 2000, São Paulo. Anais do ENEGEP/2000, 8f. SILVA, Luis Roberto. Customização em Massa nos serviços de empresas de varejo. 2001. 130f. Dissertação (Mestrado em Engenharia de Produção) - Centro Tecnológico, Curso de Pós-Graduação em Engenharia de Produção, Universidade Federal Fluminense, Niterói. 2001.

SILVA, Luis Roberto. Customização em massa nos serviços de empresas de varejo.2001. 130f. Dissertação (Mestrado em Engenharia de Produção) - Centro Tecnológico, Curso de Pós-graduação em Engenharia de Produção, Universidade Federal Fluminense, Niterói.2001.

\subsection{OBRAS REFERENCIADAS}

BOGADO, Savio D.C. Estado da arte do uso competitivo de tecnologia de informação no setor de bebidas nãoalcoólicas (um estudo de caso de sete empresas à luz dos modelos de estabilidade dinâmica e da cadeia de valor).2003. 242 f. Dissertação (Mestrado em Engenharia de Produção) - Centro Tecnológico, Curso de Pós-graduação em Engenharia de Produção, Universidade Federal Fluminense, Niterói. 2003. 
FERRAZ, João Carlos; COUTINHO, Luciano. Estudo da Competitividade da Industria Brasileira - ECIB.Brasília/DF: Ministério da Ciência e Tecnologia (MCT), na Internet no site WWW.mct.org.rr. Acesso: Julho de 2002.

FREITAS, Henrique; LESCA, $\mathrm{H}$. Competitividade empresarial na era da informação. São Paulo; REVISTA DE ADMINISTRAÇÃO DA USP, v.27, n.3, Julho/Setembro 1992 p.92-102

LUNA, Sergio Vasconcelos. Planejamento de pesquisa: uma introdução. São Paulo: EDUC, 1997.

MCFARLAN, F.W. Information Technology changes the way you compete. HARVARD BUSINESS REVIEW, v.62, pp. 98-103, May/June, 1984.

MCKENNEY, J.L.; MCFARLAN, F.W. The Information Archipelago-Maps and Bridges. HARVARD BUSINESS REVIEW, Sep/OCT., 1982, p.109.

PORTER, Michael E.; MILLAR, Victor. How information gives you competitive advantage. HARVARD BUSINESS REVIEW, Boston/USA, July/Aug., v.63, n.4, pp. 149-160, 1985

QUINTELLA, Heitor L.M.M. Fatores Humanos e Tecnológicos da Competitividade: Projeto de Pesquisa. UFF - Centro Tecnológico, 1999. 